\title{
Characterization of aerosol and assessment of the risk of transmission of SARS-COV-2 VIRUS in a natural thermal cave
}

\author{
Mauro Scungio ${ }^{1, *}$, and Giulia Parlani $^{1}$ \\ ${ }^{1}$ Department of Economics, Engineering, Society and Business Organization (DEIM), University of \\ Tuscia, Via del Paradiso 47, Viterbo, Italy
}

\begin{abstract}
Thermal caves represent an environment characterized by unique chemical-physical properties, often used by customers for treatment and care of musculoskeletal, respiratory and skin diseases. The recent pandemic caused by COVID-19 has imposed the need to investigate the potential transmission scenario of $S A R S-C o V-2$ virus also in such atypical and poorly studied indoor environments. This research work was carried out inside a natural thermal cave located in Italy where a waterfall of sulfur-sulfatebicarbonate-alkaline earth mineral thermal water creates a warm-humid environment with $100 \%$ humidity and $48^{\circ} \mathrm{C}$ temperature. A characterization of the aerosol was carried out in terms of number, surface area and mass, as well as particle size distributions. The physical characteristics of the aerosol were measured inside the natural thermal cave and in other immediately adjacent areas in two different days and in two distinct moments by means of an optical spectrometer. The data obtained showed a predominance of particles with a diameter greater than $8 \mu \mathrm{m}$, associated with a low ability of penetration in the human respiratory system. Subsequently, through a model recently proposed in scientific literature, it was evaluated the airborne transmission risk of SARS-CoV-2 inside the cave by quantifying the probability of infection due to exposure in a microenvironment in presence of a SARS-CoV-2 infected subject. The infection risk was evaluated for different scenarios obtained combining parameters such as physical, breathing or talking activities of the occupants, simultaneous or nonsimultaneous access to the cave and mechanical ventilation activated or nonactivated. In terms of the risk of SARS-CoV-2 infection, evaluated under the hypotheses of the model, it was highlighted the decisive effect of the mechanical ventilation system on the risk of contagion: for all the hypothesized scenarios, there is a substantial reduction in the risk of contagion considering the ventilation system active. Furthermore, the adoption of social distancing measures such as non-simultaneous access to the cave makes the risk of contagion extremely low, according to the assumptions underlying the model, even with the mechanical ventilation system not active.
\end{abstract}

\footnotetext{
${ }^{*}$ Corresponding author: mauro.scungio@unitus.it
} 


\section{Introduction}

Thermal facilities, such as caves, swimming pools and SPAs, represent an environment characterized by unique chemical-physical properties, such as the high concentration of mineral salts and dissolved gases, peculiar temperatures and $\mathrm{pH}$. Depending on the content of specific elements in the thermal waters, such as bicarbonates, calcium, sulphur, sulphates, chlorides, radon, iron, magnesium, potassium, lithium, arsenic and silica, different therapeutic effects for musculoskeletal, respiratory and skin diseases can be observed [1].

The recent pandemic caused by COVID-19 has imposed the need to investigate the potential transmission scenario of $S A R S-C o V-2$ virus also in such atypical and poorly studied indoor environments, where an in-depth analysis of the aerosol concentrations and dimensional distributions are essential in order to monitor the spread of the virus. While some studies and models have been proposed to estimate the airborne risk of transmission in public environments such as hospitals or restaurants, characterized by mild climatic conditions in terms of temperature and relative humidity, thermal environments have been poorly investigated; furthermore, the specific properties of these environments prevent their treatment and the application of conventional disinfection procedures in order to preserve their health benefits during balneotherapy or other treatments.

The main route of transmission of the SARS-CoV-2 virus to humans occurs predominantly via the respiratory route, by means of both small and large droplets [2]. The risk of transmission is dependent on different factors: droplet properties, indoor airflow and virus characteristics. Droplet size influences both the deposition mechanisms and the extent of penetration in the human respiratory systems. Small droplets $(1-5 \mu \mathrm{m})$ can remain suspended in the air for many hours and penetrate up to the alveoli, while larger droplets have a high sedimentation rate and deposit in the upper respiratory tract. Moreover, being droplets constituted mostly of water associated with an aerosol-size nucleus, the evaporation kinetics, influenced by relative humidity and air temperature, affect their lifetime and deposition.

The ventilation systems and convection currents play a critical role in indoor environments: if appropriate, they can promote the removal of exhaled virus-laden air, thus lowering the airborne viral concentration; in case of inefficient or obstructed airflow, they can disperse the aerosol over a large area, with the potential to increase the risk of transmission to other occupants [4-6].

For risk management in the initial phase of reopening of the thermal structures, some protocols and self-control plans have been developed but the development of new scientific evidence on this is desirable.

This work was carried out inside a natural thermal cave located in Italy where a waterfall of sulfur-sulfate-bicarbonate-alkaline earth mineral thermal water creates a warm-humid environment with $100 \%$ humidity and $48^{\circ} \mathrm{C}$ temperature. A characterization of the aerosol was carried out with the aim of estimating personal exposure to aerosol concentrations in terms of number, surface area and mass, as well as particle size distributions. The final purpose was the identification of specific thresholds and alert values to support the development of a Risk Assessment Plan in thermal caves.

\section{Materials and methods}

\subsection{Aerosol physical characteristics measurements}

In this work the physical characteristics of the aerosol were measured by means of an Optical Particle Sizer (OPS model 3330, TSI, Minnesota, USA). The OPS was calibrated prior the measurement campaign by means of tests made in the European Accredited Laboratory of 
Industrial Measurements (LAMI) at the University of Cassino and Southern Lazio (Italy) using Polystyrene Latex (PSL) spheres.

In the thermal cave, the aerosol samplings were repeated in two different days and in two distinct moments: at the morning before the first entrance to the cave and at the evening after the last entrance. Each measurement represented the average of several samplings lasting 100 seconds and was related to a number, surface area and mass concentration as well as size distributions of particles per unit volume of sampled air.

\subsection{SARS-CoV-2 transmission risk evaluation}

To evaluate the airborne transmission risk of SARS-CoV-2 inside the cave, a model recently proposed was adopted [2]. The model is able to quantify the probability of infection due to exposure in a microenvironment in presence of a SARS-CoV-2 infected subject, by means of a four step approach: (i) evaluation of the quanta emission rate (quanta is defined as the dose of airborne droplet nuclei required to cause infection in $63 \%$ of susceptible persons) [1]; (ii) evaluation of the exposure to quanta concentration in the microenvironment; (iii) evaluation of the dose of quanta received by an exposed susceptible subject; and (iv) estimation of the probability of infection on the basis of a dose-response model. These four steps are briefly described in hereafter, referring to the original papers for more details $[2,3]$.

The quanta emission rate $\left(\mathrm{ER}_{\mathrm{q}}\right.$, quanta $\left.\mathrm{h}^{-1}\right)$ is evaluated as (first step):

$$
E R_{q}=c_{v} \cdot c_{i} \cdot I R \cdot V_{d}=c_{v} \cdot \frac{1}{C_{R N A} \cdot C_{P F U}} \cdot I R \cdot V_{d}
$$

where $\mathrm{c}_{\mathrm{v}}$ (RNA copies $\mathrm{mL}^{-1}$ ) is the viral load in the exhaled droplets, $\mathrm{c}_{\mathrm{i}}$ (quanta RNA copies $\left.{ }^{-1}\right)$ is a conversion factor, IR is the inhalation rate $\left(\mathrm{m}^{3} \mathrm{~h}^{-1}\right)$, and $\mathrm{V}_{\mathrm{d}}$ is the exhaled droplet volume concentration $\left(\mathrm{mL} \mathrm{m}^{-3}\right)$, which depends on the repiratory activity.

The quanta concentration at time $t$ to which a susceptible subject is exposed in an indoor environment is based on a mass balance and can be evaluated as (second step):

$$
n\left(t, E R_{q}\right)=n_{0} \cdot e^{-I V R R \cdot t}+\frac{E R_{q} \cdot I}{I V R R \cdot V} \cdot\left(1-e^{-I V R R \cdot t}\right)
$$

where IVRR $\left(\mathrm{h}^{-1}\right)$ represents the infectious virus removal rate, $\mathrm{n}_{0}$ represents the initial quanta concentration, $\mathrm{I}$ is the number of infectious subjects, $\mathrm{V}$ is the volume of the indoor environment, and $\mathrm{ER}_{\mathrm{q}}$ is the quanta emission rate for the virus under investigation. IVRR depends on three mechanisms: air exchange rate via ventilation, particle deposition on surfaces and viral inactivation. There are three important hypotheses on the quanta concentration calculation: i) the infectious virus removal rate is constant, ii) the latent period of the disease is longer than the time scale of the model, and iii) the droplets are instantaneously and evenly distributed in the indoor environment.

The dose of quanta received by an exposed susceptible subject to the quanta concentration, for a certain time interval $(\mathrm{T})$, is evaluated as (third step):

$$
D_{q}\left(E R_{q}\right)=I R \int_{0}^{T} n(t) d t
$$

The probability of infection is calculated by an exponential dose-response model as (fourth step):

$$
P_{I}=1-e^{-D_{q}}
$$

Finally, the individual infection risk $(\mathrm{R})$ of an exposed subject is calculated as the product of the probability of infection and the probability of occurrence of the specific $\mathrm{ER}_{\mathrm{q}}$ value, 
while the basic reproduction number $\left(\mathrm{R}_{0}\right)$ is evaluated by multiplying the individual infection risk by the number of exposed susceptible individuals $[5,6]$.

\subsection{Model parameters and hypothesized scenarios for the risk of infection transmission inside the cave}

The parameters set as input data of the model are: volume of the cave (V), total infectious viral removal rate (IVRR), which is the sum of the number of air exchanges per hour (AER), the deposition rate of particles on the surfaces $(k)$ and rate of viral inactivation $(\lambda)$, initial concentration of quanta $\left(\mathrm{n}_{0}\right)$ and total time of occupation of the cave $(\mathrm{t})$.

The volume of the cave was calculated directly from measurements carried out on site and is equal to $34 \mathrm{~m}^{3}$ while the number of hourly changes of air was obtained from the technical specifications of the ventilation system installed $\left(260 \mathrm{~m}^{3} \mathrm{~h}^{-1}\right)$ plus $0.2 \mathrm{~h}^{-1}$ to consider the infiltrations deriving from the partial opening of the skylight, for a total of AER equal to $7.78 \mathrm{~h}^{-1}$. The deposition rate of the particles (k) was set equal to $0.24 \mathrm{~h}^{-1}$ as proposed by Buonanno et al. [2] which calculated the deposition rate as the ratio between the sedimentation rate of particles whose diameter is greater than $1 \mu \mathrm{m}$ (approximately $1.0 \times 10^{-4}$ $\mathrm{m} \mathrm{s}^{-1}$ ) as measured by Chatoutsidou and Lazaridis [7] and the height of the emission source $(1.5 \mathrm{~m})$. The viral inactivation rate $(\lambda)$ was set equal to $0.63 \mathrm{~h}^{-1}$ based on the value of the SARS-CoV-2 half-life (1.1 hours) detected as follows [8]:

$$
\lambda\left(h^{-1}\right)=\frac{0.693}{t_{1 / 2}}
$$

Finally, the initial quanta concentration $\left(\mathrm{n}_{0}\right)$ and the total occupation time of the cave $(\mathrm{t})$ were set equal to zero and 8 hours, respectively. All the parameters are shown in detail in Table 1.

Table 1. Summary of the input data used in the model for calculating the risk of infection inside the thermal cave.

\begin{tabular}{cccccc}
\hline $\mathbf{V}\left(\mathbf{m}^{\mathbf{3}}\right)$ & $\mathbf{A E R}\left(\mathbf{h}^{-\mathbf{1}}\right)$ & $\mathbf{k}\left(\mathbf{h}^{-\mathbf{1}}\right)$ & $\boldsymbol{\lambda}\left(\mathbf{h}^{\mathbf{- 1}}\right)$ & $\left.\mathbf{n} \mathbf{\text { nquanta}} / \mathbf{m}^{\mathbf{3}}\right)$ & $\mathbf{t}(\mathbf{m i n})$ \\
\hline 34 & 7.78 & 0.24 & 0.63 & 0 & 480 \\
\hline
\end{tabular}

As regards the hypothesized scenarios, these have been implemented by setting the following parameters: time of entry and exit from the cave and the emission rate of quanta of infectious subjects $\left(E_{\mathrm{q}}\right)$; time of entry and exit from the cave and inhalation rate (IR) of the susceptible subject. Four scenarios have been hypothesized: the first (S1) involves the consecutive entry into the cave of two infectious subjects, for a residence time of 15 minutes for each, and subsequent entry into the cave of the susceptible subject, with a residence time of the latter equal to 15 minutes; the second (S2) involves the entry and stay of two infectious subjects and a susceptible subject simultaneously for 15 minutes. In the first scenario (S1) all the occupants were at rest (seated) and characterized only by respiratory activity, while in the second (S2) the occupants were standing and talking to each other. Both S1 and S2 scenarios have been implemented considering the ventilation system in operation and semi-opened skylight $\left(\mathrm{AER}=7.78 \mathrm{~h}^{-1}\right)$. Starting for S1 and S2, two additional scenarios were considered: S3 (same of S1 without the ventilation system active) and S4 (same of S2 without the ventilation system active). For S3 and S4, however, an AER value of $0.2 \mathrm{~h}^{-1}$ was imposed to consider infiltrations through the doors. Details of the simulated scenarios are reported in Table 2. 
Table 2. Details of the exposure scenarios in the thermal cave implemented in the model $\left(\mathrm{t}_{\mathrm{in}}=\right.$ time of entry into the cave, $t_{\text {out }}=$ time of exit from the cave, $E_{\mathrm{q}}=$ emission rate of quanta, $\mathrm{IR}=$ inhalation rate, $\mathrm{AER}=$ air exchange rate).

\begin{tabular}{ccccccccccc}
\hline Scenario & \multicolumn{3}{c}{ Infectious subject 1 } & \multicolumn{3}{c}{ Infectious subject 2 } & \multicolumn{3}{c}{ Susceptible subject } \\
\hline & $\begin{array}{c}\mathbf{t}_{\text {in }} \\
(\mathbf{m i n})\end{array}$ & $\begin{array}{c}\mathbf{t}_{\text {out }} \\
(\mathbf{m i n})\end{array}$ & $\begin{array}{c}\mathbf{E R}_{\mathbf{q}} \\
\left(\mathbf{q u a n t a} \mathbf{h}^{-\mathbf{1}}\right)\end{array}$ & $\begin{array}{c}\mathbf{t}_{\text {in }} \\
(\mathbf{m i n})\end{array}$ & $\begin{array}{c}\mathbf{t}_{\text {out }} \\
(\mathbf{m i n})\end{array}$ & $\begin{array}{c}\mathbf{E R}_{\mathbf{q}} \\
\left(\mathbf{q u a n t a} \mathbf{h}^{-\mathbf{1}}\right)\end{array}$ & $\begin{array}{c}\mathbf{t}_{\text {in }} \\
(\mathbf{m i n})\end{array}$ & $\begin{array}{c}\mathbf{t}_{\text {out }} \\
(\mathbf{m i n})\end{array}$ & $\begin{array}{c}\mathbf{I R} \\
\left(\mathbf{m}^{\mathbf{3}} \mathbf{h}^{\mathbf{1}}\right)\end{array}$ & $\begin{array}{c}\text { AER } \\
\left(\mathbf{h}^{\mathbf{1}}\right)\end{array}$ \\
\hline $\mathbf{S 1}$ & 0 & 15 & 2.0 & 15 & 30 & 2.0 & 30 & 45 & 0.49 & 7.78 \\
\hline $\mathbf{S 2}$ & 0 & 15 & 11.4 & 0 & 15 & 11.4 & 0 & 15 & 0.54 & 7.78 \\
\hline $\mathbf{S 3}$ & 0 & 15 & 2.0 & 15 & 30 & 2.0 & 30 & 45 & 0.49 & 0.2 \\
\hline $\mathbf{S 4}$ & 0 & 15 & 11.4 & 0 & 15 & 11.4 & 0 & 15 & 0.54 & 0.2 \\
\hline
\end{tabular}

\section{Results and discussion}

\subsection{Physical characteristics of the aerosol inside the thermal cave}

This section contains the results of aerosol measurements in the different areas sampled. The data are shown as size distributions (Fig. 1) and average concentrations (Table 3) in terms of number $(\mathrm{N})$, surface area (SA) and mass $(\mathrm{M})$ of particles per unit volume of sampled air.

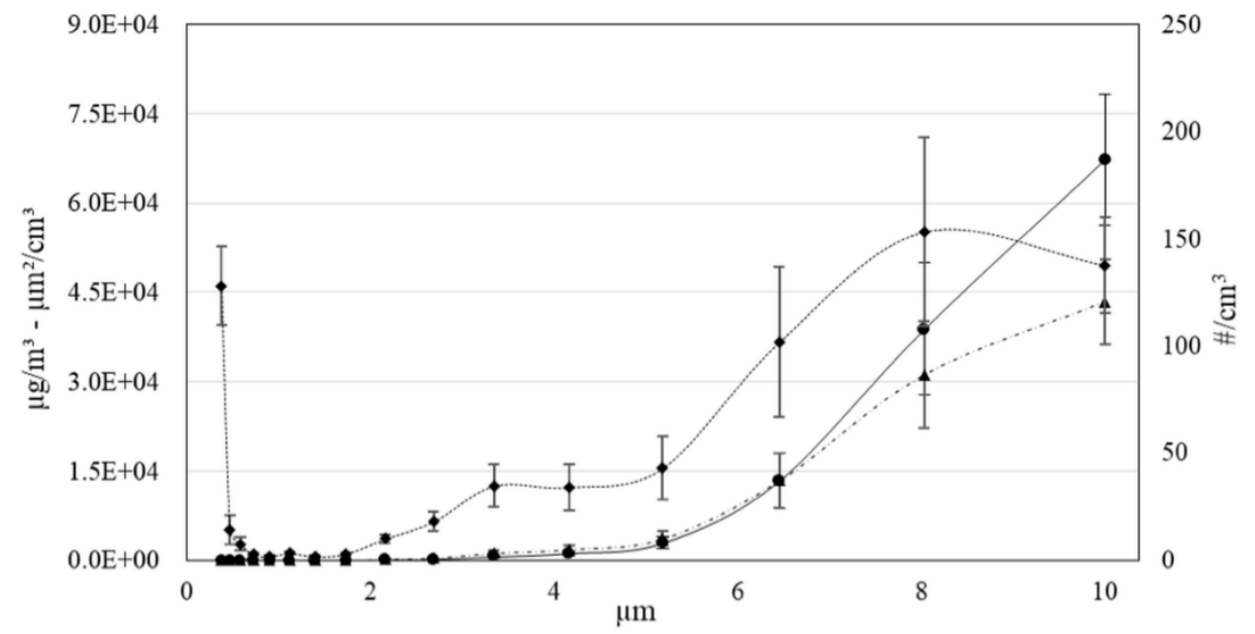

Fig. 1. Particle size distributions of the aerosol measured inside the cave in terms of mass $(M$, in $\mu \mathrm{g}$ $\mathrm{m}^{-3}$, solid line with circles), surface area (SA, in $\mu \mathrm{m}^{2} \mathrm{~cm}^{-3}$, dashed-dotted line with triangles) and number $\left(\mathrm{N}\right.$, in $\# \mathrm{~cm}^{-3}$, dotted line with diamonds) of particles per volume unit of sampled air. The error bars represent the standard deviation in the measured data on each channel.

Table 3. Average concentrations of the aerosol measured inside the cave in terms of number of particles per $\mathrm{cm}^{3}$ of air $(\mathrm{N})$, surface area of particles per $\mathrm{cm}^{3}$ of air (SA) and mass of particles per $\mathrm{m}^{3}$ of air (M).

\begin{tabular}{|c|c|c|}
\hline & Average total concentration & Standard deviation \\
\hline $\mathbf{N}\left(\# \mathbf{c m}^{-3}\right)$ & $6.9 \times 10^{2}$ & $1.3 \times 10^{2}$ \\
\hline $\mathrm{SA}\left(\mu \mathbf{m}^{2} \mathbf{c m}^{-3}\right)$ & $9.5 \times 10^{4}$ & $2.2 \times 10^{4}$ \\
\hline $\mathbf{M}\left(\mu \mathbf{g ~ m}^{-3}\right)$ & $1.2 \times 10^{5}$ & $2.7 \times 10^{4}$ \\
\hline
\end{tabular}


Since no significant changes in the aerosol characteristics were observed between the two days of measurements, and between morning and evening for each day, the data are shown as average values with the relative standard deviation among all the measurements carried out.

From the analysis of the data relating to the physical characteristics of the aerosol measured inside the thermal cave, the dimensional distribution in terms of number of particles results as tri-modal, with the first mode located around $0.3 \mu \mathrm{m}$, a second one around $3 \mu \mathrm{m}$ and the last one around $8 \mu \mathrm{m}$, as showed in Fig. 1. It should be noted, anyway, that the two modes that mainly characterize the size distribution in terms of number are the first one $(0.3$ $\mu \mathrm{m})$ and the last one $(8 \mu \mathrm{m})$, while the mode at $3 \mu \mathrm{m}$ results less pronounced. The size distributions in terms of surface area and mass of particles, on the contrary, resulted unimodal with mode at $10 \mu \mathrm{m}$. From the analysis of the data, it can be stated that the dimensional distribution in number highlights the presence in the cave of a significant fraction of particles whose dimensions were below the detection threshold of the spectrometer (sub-micrometric particles) even if the fraction of particles with mode around $8 \mu \mathrm{m}$ was predominant. On the other hand, both the distributions in terms of surface area and mass of particles were characterized with a mode totally shifted to higher particle diameters.

Summarising, the analysis of the measured data indicated the presence in the cave of a significant fraction of sub-micrometric particles and a predominant fraction of supermicrometric particles contributing to the surface area and mass concentration. These supermicrometric particles consisted, presumably, of water droplets, while the sub-micrometric particles may consist of the solid nuclei, resulting from the evaporation of the volatile fraction. As the aerosol measured in the thermal cave was mainly composed of supermicrometric particles, it can be concluded that these are the most deposited in the respiratory system; so the use of the described risk assessment model of infection transmission can be considered particularly suitable and reliable.

\subsection{Aerosol and human respiratory system}

Starting from the size distributions of the aerosol measured in the cave and using appropriate deposition curves (ICRP, 1994), the lung-deposited fraction of inhaled particles was calculated. The summary of the concentration in terms of number, surface area and mass of particles deposited in the respiratory system is reported in Table 4 while the deposition curves are showed in Fig. 2.

Table 4. Particle concentration in terms of number $(\mathrm{N})$, surface area (SA) and mass $(\mathrm{M})$ of particles deposited in the two regions of the respiratory system per unit of volume of inhaled air inside the cave (adults at rest, male - female, $\mathrm{ALV}=$ alveolar region, $\mathrm{TB}=$ tracheobronchial region).

\begin{tabular}{|c|c|c|c|c|c|c|}
\hline & \multicolumn{3}{|c|}{ Male } & \multicolumn{3}{|c|}{ Female } \\
\hline & ALV & TB & $\begin{array}{l}\text { Total } \\
\text { male }\end{array}$ & ALV & TB & $\begin{array}{r}\text { Total } \\
\text { female }\end{array}$ \\
\hline $\mathbf{N}\left(\# \mathbf{c m}^{-3}\right)$ & 68.02 & 25.84 & 93.86 & 59.46 & 28.37 & 87.83 \\
\hline $\mathbf{S A}\left(\mu \mathbf{m}^{2} \mathbf{c m}^{-3}\right)$ & $6.37 \times 10^{3}$ & $3.12 \times 10^{3}$ & $9.49 \times 10^{3}$ & $5.90 \times 10^{3}$ & $3.37 \times 10^{3}$ & $9.27 \times 10^{3}$ \\
\hline $\mathbf{M}\left(\mu \mathrm{g} \mathrm{m}^{-3}\right)$ & $7.85 \times 10^{3}$ & $4.00 \times 10^{3}$ & $1.18 \times 10^{4}$ & $7.30 \times 10^{3}$ & $4.29 \times 10^{3}$ & $1.16 \times 10^{4}$ \\
\hline
\end{tabular}



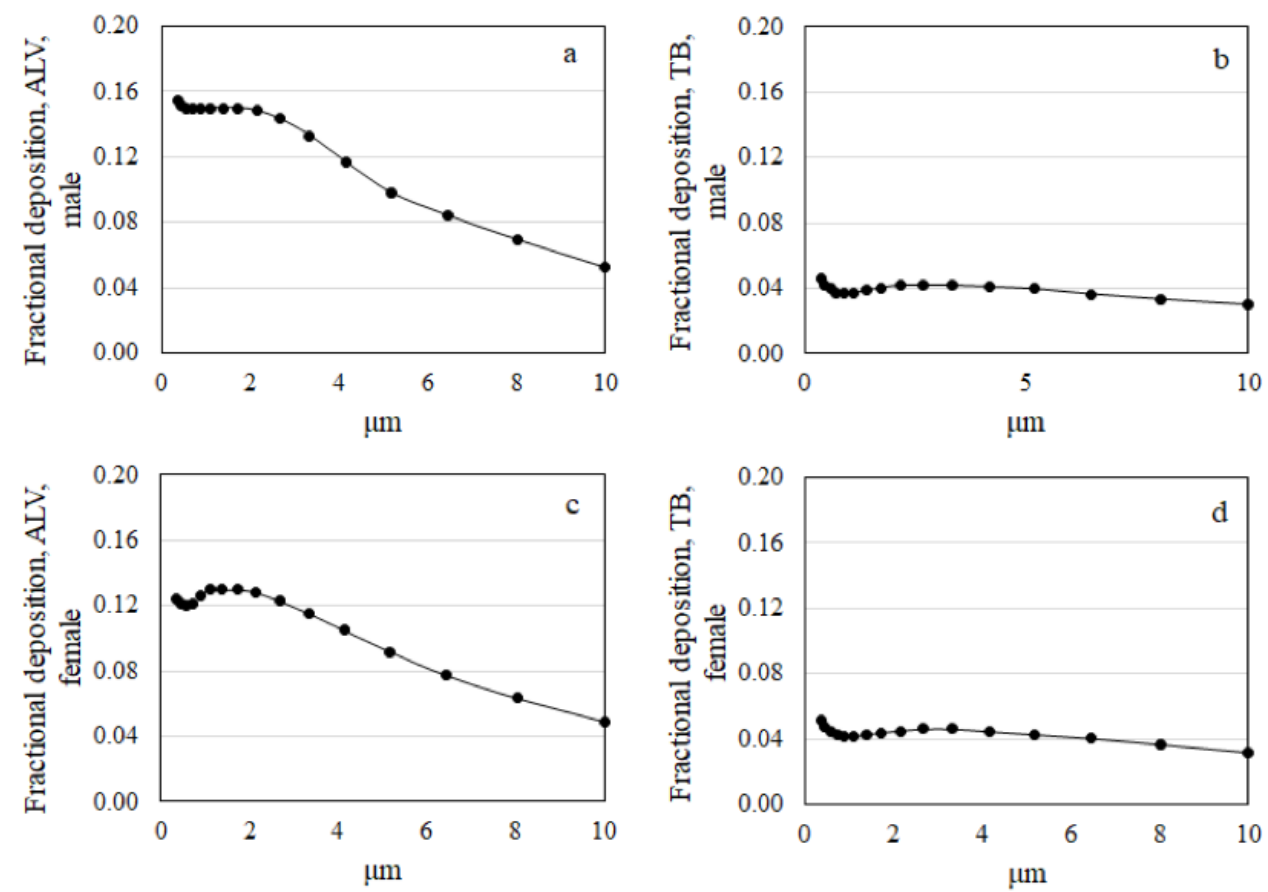

Fig. 2. Deposition curves of particles, relative to the alveolar (ALV) and tracheobronchial (TB) regions of the respiratory system specific for adult women and men at rest (seated) who breathe mainly through the nose, a typical situation of the people who occupy the thermal cave.

\subsection{Risk of infection transmission inside the thermal cave}

Based on the described methodology, the risk of SARS-CoV-2 transmission inside the cave is in hereafter reported for the susceptible occupant (relative to his residence time) and for the susceptible persons (i.e., relative to the total time of occupation). The following parameters are discussed:

- Exposure time $\left(\mathrm{t}_{\exp }\right)$ : the exit time minus the entry time in minutes for the susceptible subject and the total time of occupation for continuous occupation.

- Individual infection risk $(\mathrm{R})$ : the percentage probability of individual infection for the exposure to the quanta concentration profile integrated over the exposure time.

- Exposure time for $1 \%$ and $0.1 \%$ risk $\left(t_{\exp , 1}\right.$ and $\left.t_{\text {exp,0.1 }}\right)$ : the exposure time in minutes associated with a $1 \%$ and $0.1 \%$ probability of infection in order to consider different cohorts of individuals (younger-healthier individuals and weaker-older people, respectively).

- Maximum occupation of the cave for $\mathrm{R}_{0}<1 \quad\left(\mathrm{O}_{\max , \mathrm{R}<1}\right)$ : the maximum number of occupants theoretically allowed in the cave for the exposure time and the quanta concentration profile of the designated scenario in order to keep the base reproduction number $\left(\mathrm{R}_{0}\right)$ below 1 .

In Table 5 the symbol "greater than" means that the susceptible subject or the continuous occupation does not exceed the $0.1 \%$ or $1 \%$ risk threshold. The results of the simulations are reported in the table below: 
Table 1. Simulation results for all the hypothesized scenarios referred to both the conditions of individual risk (susceptible subject) and continuous occupation of the thermal cave ( $t_{\text {exp }}$ : exposition time; R: individual infection risk; texp,0.1: exposure time for for $0.1 \%$ risk; texp, 1 : exposure time for $1 \%$ risk; $\mathrm{O}_{\max , \mathrm{R} 0<1}$ : maximum occupation of the cave for $\mathrm{R}_{0}<1$, AER: air exchange ratio).

\begin{tabular}{|c|c|c|c|c|c|c|c|c|c|c|c|}
\hline \multirow[b]{2}{*}{ Scenario } & \multirow[b]{2}{*}{$\begin{array}{c}\mathbf{t}_{\exp } \\
(\min )\end{array}$} & \multicolumn{3}{|c|}{ Susceptible subject } & \multicolumn{7}{|c|}{ Continuous occupation } \\
\hline & & $\begin{array}{l}\mathbf{R} \\
(\%)\end{array}$ & $\begin{array}{l}\mathbf{t}_{\text {exp, }, 0.1} \\
(\mathrm{~min})\end{array}$ & $\begin{array}{l}t_{\text {exp, } 1} \\
(\min )\end{array}$ & $\mathbf{O}_{\max , \mathbf{R} 0<1}$ & $\begin{array}{c}\mathbf{t}_{\exp } \\
(\mathbf{m i n})\end{array}$ & $\mathbf{R}$ & $\begin{array}{l}\mathbf{t}_{\text {exp }, 0.1} \\
(\min )\end{array}$ & $\begin{array}{l}\mathbf{t}_{\text {exp, } \mathbf{1}} \\
(\min )\end{array}$ & $\mathbf{O}_{\max , \mathbf{R} 0<1}$ & $\begin{array}{r}\text { AER } \\
\left(h^{-1}\right)\end{array}$ \\
\hline S1 & 15 & 0.01 & $>15$ & $>15$ & 2742 & 480 & 0.02 & $>480$ & $>480$ & 605 & 7.78 \\
\hline S2 & 15 & 0.1 & $>15$ & $>15$ & 156 & 480 & 0.15 & 15 & $>480$ & 96 & 7.78 \\
\hline S3 & 15 & 0.04 & $>15$ & $>15$ & 386 & 480 & 0.20 & 54 & $>480$ & 75 & 0.2 \\
\hline S4 & 15 & 0.16 & 11 & $>15$ & 91 & 480 & 1.21 & 11 & 103 & 12 & 0.2 \\
\hline
\end{tabular}

The analysis of the data reported in Table 5 allowed to characterize the cave in terms of risk of infection under the assumptions of the model and for the considered scenarios, the results obtained for various scenarios are reported in Fig. 3. First of all, considering the reported data, it should be highlighted the determining effect of the mechanical ventilation system on the risk of contagion: for all the hypothesized scenarios, there is a substantial reduction in the risk of contagion considering the ventilation system active, both for the individual susceptible subject in the 15 minutes of stay and for continuous occupation of the cave. This effect is made even more evident by the parameter relating to the maximum occupation of the cave (continuous occupation) which changes from the theoretical value of 75 to that of 605 (with ventilation system deactivated, S3, and activated, S1, respectively), and from 12 to 96 (with ventilation system deactivated, S4, and activated, S2, respectively). It should be highlighted, however, the purely theoretical role, albeit significant, of this parameter: it is unlikely, in fact, the simultaneous presence of more occupants than the surface of an indoor environment would allow.

Furthermore, from the analysis of the results, it is evident that the adoption of social distancing measures such as non-simultaneous access to the cave (S1) makes the risk of contagion extremely low, according to the assumptions of the model. even with the mechanical ventilation system not active. A condition that presents a relatively high risk for the single susceptible subject is that relating to the modelled scenario with the ventilation system not active and the presence of two infectious subjects and one susceptible subject at the same time in the cave talking to each other (S4). In this case, in fact, assuming a probability of infection of $0.1 \%$, the maximum exposure time is less than the 15 -minute of residence time for the susceptible subject (11 minutes). On the contrary, for scenario 1 with the ventilation system not active (S3), the maximum exposure time is less than the maximum time (54 minutes out of 480), highlighting, once again, the fundamental role of the mechanical ventilation system. Finally, from the analysis of Fig. 3, it can be seen that the mechanical ventilation system active results in a sudden reduction on the quanta concentration in the cave (solid lines in Fig. 3) that reaches a zero value after about 75 minutes for both $\mathrm{S} 1$ and $\mathrm{S} 3$ scenarios and in a consequent reduction of the risk of contagion (both individual risk, dashed lines, and continuous risk, dotted lines) and increase in maximum occupancy (dashed-dotted lines). On the contrary, with the ventilation system not active, the quanta concentration reaches zero value after about 460 minutes for scenario 2 while for the worst hypothesized scenario (S4), the quanta concentration still remains greater than zero after the whole simulation period (480 minutes).

The results obtained from the model for each considered scenario are reported in Fig. 3 as function of time. The reported time-dependent parameters are organised as follows:

- Quanta concentration in the cave in quanta $/ \mathrm{m}^{3}$ (solid lines). 
- Individual risk of infection for the susceptible subject (dashed lines).

- Individual infection risk for a subject who continuously occupies the cave for the entire simulated time of 8 hours (dotted lines).

- Maximum theoretical occupancy of the cave to maintain $\mathrm{R}_{0}<1$ (dashed-dotted lines).
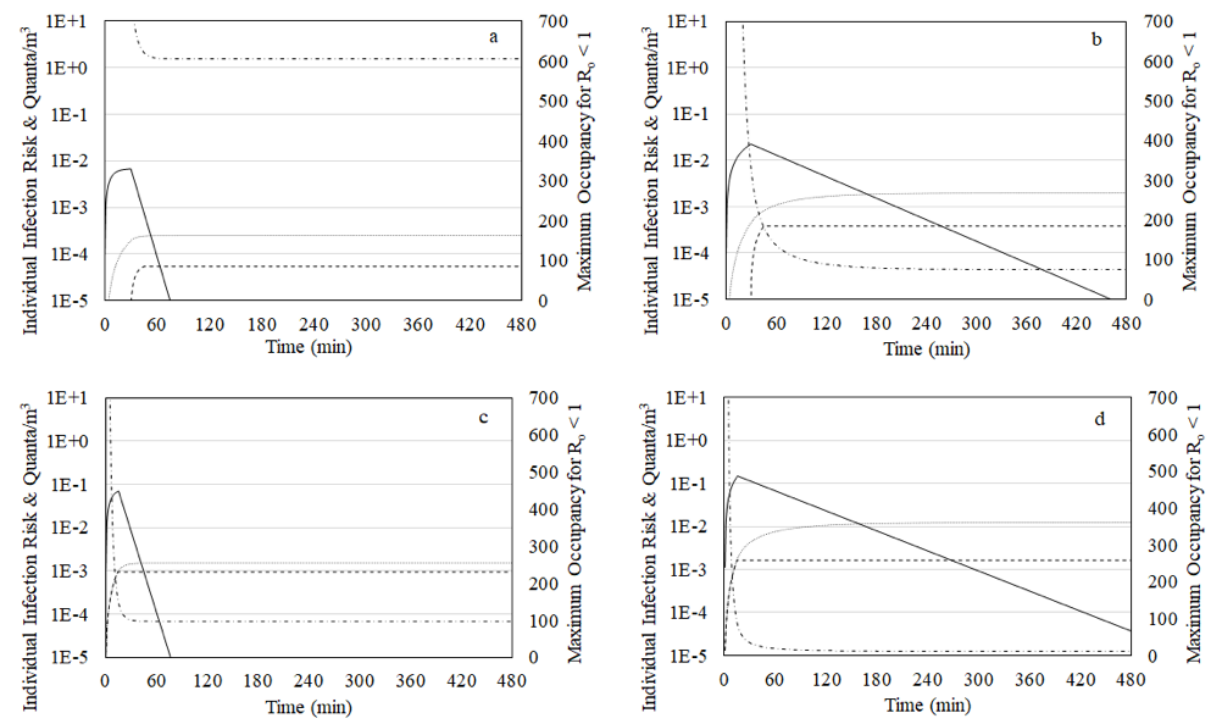

Fig. 3. Simulation results time course: scenario 1 (S1, ventilation system in operation, a); scenario 2 (S2, ventilation system not in operation, b); scenario 3 (S3, ventilation system in operation, c) and scenario 4 (S4, ventilation system not in operation, d). Quanta concentration (solid lines); continuous risk (dotted lines); individual infection risk (dashed lines) and maximum occupancy (dashed-dotted lines).

The authors want to highlight the main limitation of the model which lies in the "box model" approach [3], according to which the air is perfectly mixed in the cave and, consequently, the quanta concentration over time becomes uniform in space. According to this approach, susceptible individuals are therefore exposed to the same quanta concentration regardless of their position in the cave. Differences in exposure risk between susceptible occupants are therefore reduced to a function of the duration of exposure rather than their position in the cave. In the light of these simplifying hypotheses, the accuracy of the model depends on the spatial scale analysed. In general, the smaller the enclosed space, the more the air is mixed and therefore the closer the results are to reality.

\section{Conclusions}

In this paper the physical characteristics of the aerosol inside a thermal cave located in the middle Italy were analysed in terms of particle mass, number and surface area concentrations, as well as size distributions. In addition, on the basis of the observed physical characteristics of the aerosol particles, a model for the estimation of the infection transmission risk of SARSCoV-2 was applied to four different exposure scenarios of the occupants of the thermal cave.

The physical characterization of the aerosol revealed a tri-modal dimensional distribution in terms of number of particles, with the first mode located around $0.3 \mu \mathrm{m}$, a second one around $3 \mu \mathrm{m}$ and the last one around $8 \mu \mathrm{m}$. The size distributions in terms of surface area and mass of particles, on the contrary, resulted unimodal with mode at $10 \mu \mathrm{m}$. The analysis of the measured data indicated the presence in the cave of a significant fraction of sub- 
micrometric particles, whose main contribution lies in the number concentration, and a predominant fraction of super-micrometric particles contributing to the surface area and mass concentration. These super-micrometric particles consisted, presumably, of water droplets, while the sub-micrometric particles may consist of the solid nuclei, resulting from the evaporation of the volatile fraction, which remain suspended in air.

In terms of the risk of SARS-CoV-2 infection, evaluated under the hypotheses of the model, it is highlighted the decisive effect of the mechanical ventilation system on the risk of contagion: for all the hypothesized scenarios, there is a substantial reduction in the risk of contagion considering the ventilation system active. Furthermore, the adoption of social distancing measures such as non-simultaneous access to the cave makes the risk of contagion extremely low, according to the assumptions underlying the model, even with the mechanical ventilation system not active.

\section{Funding and acknowledgments}

This work has been carried out within the framework of COVID-19 activities of ISUM/CINTEST group and has received funding from both, University of Tuscia, Viterbo (Italy) under "Action for research activities related to COVID-19emergency" Rector note $\mathrm{n}^{\circ}$ 5445, 05-05-2020, and from Terme dei Papi (Viterbo, Italy) within the study "Characterization of Bioaerosol in the Thermal Cave of Terme dei Papi and effect of environmental conditions in the decay and transmission of biological agents".

\section{References}

1. N. Altman, Healing springs: the ultimate guide to taking the waters. Inner Traditions/Bear \& Co (2000)

2. G. Buonanno, L.Stabile, L. Morawska, Estimation of Airborne Viral Emission: Quanta Emission Rate of SARS-CoV-2 for Infection Risk Assessment (2020)

3. G. Buonanno, L. Morawska, L. Stabile, Quantitative Assessment of the Risk of Airborne Transmission of SARS-CoV-2 Infection: Prospective and Retrospective Applications. MedRxiv, https://doi.org/10.1101/2020.06.01.20118984, (2020)

4. L. Gammaitoni, M.C. Nucci, Using a Mathematical Model to Evaluate the Efficacy of TB Control Measures. Emerg. Infect. Dis. 335-342, (1997)

5. B.G. Wagner, B.J Coburn, S. Blower, Calculating the Potential for Within-Flight Transmission of Influenza A (H1N1). BMC Med. 7, 81 (2009)

6. L.D. Knibbs, L. Morawska, S.C. Bell, P. Grzybowski, Room Ventilation and the Risk of Airborne Infection Transmission in 3 Health Care Settings Within a Large Teaching Hospital. Am. J. Infect. Control 39, 866-872, (2001)

7. S.E. Chatoutsidou, M. Lazaridis, Assessment of the Impact of Particulate Dry Deposition on Soiling of Indoor Cultural Heritage Objects Found in Churches and Museums/Libraries. J. Cult. Heritage 39, 221-228, (2019) 
8. N. van Doremalen, T. Bushmaker, D.H. Morris, M.G. Holbrook, A. Gamble, B.N. Williamson, A. Tamin, J.L. Harcourt, N.J. Thornburg, S.I. Gerber, J.O. Lloyd- Smith, E. de Wit, V.J. Munster, Aerosol and Surface Stability of SARSCoV-2 as Compared with SARS-CoV-1. N. Engl. J. Med. (2020) 